\title{
Associations Between Lifestyle Factors and Reduced Kidney Function in US Older Adults: NHANES 1999-2016
}

\author{
Horng-Jinh Chang ${ }^{1}$, Kuan-Reng Lin ${ }^{1 *}$, Meng-Te Lin ${ }^{2}$ and Junn-Liang Chang ${ }^{2,3 *}$ \\ ${ }^{1}$ Department of Management Sciences, Tamkang University, Taiwan, ${ }^{2}$ Department of Pathology and Laboratory Medicine, \\ Taoyuan Armed Forces General Hospital, Taoyuan, Taiwan, ${ }^{3}$ Biomedical Engineering Department, Ming Chuan University, \\ Taoyuan, Taiwan
}

Objective: This study aimed to evaluate the associations between lifestyle factors and the estimated glomerular filtration rate (eGFR) levels in older adults by analyzing the United States National Health and Nutrition Examination Survey data (1999-2016).

Methods: A total of 10,052 eligible participants were divided into two groups: reduced eGFR group (eGFR $<60 \mathrm{ml} / \mathrm{min} / 1.73 \mathrm{~m}^{2}$ ) and normal group (eGFR $\geq 60 \mathrm{ml} / \mathrm{min} / 1.73 \mathrm{~m}^{2}$ ). The primary factors were physical activity, alcohol consumption, smoking, and comorbidities.

Results: Multivariable analysis revealed that older age, proteinuria, cardiovascular disease, diabetes, hyperuricemia, and hypertension were significantly associated with higher odds of reduced kidney function. Sufficient physical activity, current alcohol consumption, and being a current smoker were significantly associated with lower odds of reduced kidney function. However, subgroup analysis by sex revealed that the effects of proteinuria, current alcohol consumption, and sufficient physical activity were sex-specific.

OPEN ACCESS

Edited by:

Salvatore Panico,

University of Naples Federico II, Italy

${ }^{*}$ Correspondence: Kuan-Reng Lin kittylintku@gms.tku.edu.tw Junn-Liang Chang junn9liang@yahoo.com.tw

Received: 08 January 2021 Accepted: 26 May 2021 Published: 15 July 2021

Citation:

Chang H-J, Lin K-R, Lin M-T and Chang J-L (2021) Associations Between Lifestyle Factors and Reduced Kidney Function in US Older Adults: NHANES 1999-2016. Int J Public Health 66:1603966. doi: 10.3389/ijph.2021.1603966
Conclusion: Several risk and beneficial factors for reduced kidney function in adults aged 65 and above in the United States were identified, but some of them might be sex-specific. Further studies are warranted to confirm these findings in other populations and countries.

Keywords: chronic kidney disease, estimated glomerular filtration rate, exercise, kidney function, risk factors

\section{INTRODUCTION}

Populations around the world are aging as a result of healthcare advances that have reduced mortality among youth and increased longevity [1]. Accompanying the increasing older adult population, however, are the inevitable age-associated declines in organ function and biological systems, including changes in the heart and vascular system, the lungs and respiratory system, central nervous system, and cognitive function [2]. Aging itself is suggested to be a risk factor for cardiovascular disease [3]. The decline in kidney function, rather than directly associated with aging, appears to result from age-related changes in the vasculature and cardiovascular risk factors, as well as from genetic factors $[4,5]$. That is, the increased prevalence of cardiovascular disease influences the prevalence of chronic kidney disease (CKD), although it is not known to what extent compared to the effects of aging such as renal structural changes [1]. CKD is reported to affect 47 
million (14.8\%) adults in the United States (US) alone [6]; the prevalence in the global population is $11-13 \%$ [7].

CKD is diagnosed when abnormalities of kidney function or structure have been noted for 3 months or more, including an estimated glomerular filtration rate (eGFR) less than $60 \mathrm{ml}$ per minute per $1.73 \mathrm{~m}^{2}$ along with the presence of albuminuria, abnormal urine sediment, abnormal renal imaging findings, serum electrolytes, or acid-base derangements [8]. eGFR is a primary parameter for evaluating kidney function, making it a reliable biological marker for $\mathrm{CKD}$ and for monitoring fluctuations in kidney function. All individuals with an eGFR of less than $60 \mathrm{ml} / \mathrm{min} / 1.73 \mathrm{~m}^{2}$ at least twice within an interval of 90 days with or without other markers of kidney damage are said to have CKD. Risk factors for CKD may also be in evidence, including smoking, excessive alcohol consumption, obesity, hypertension, diabetes mellitus, and a history of cardiovascular disease [9].

In our previous cross-sectional study [10], we analyzed crosssectional data from the Taipei City Elderly Health Examination database to investigate associations between lifestyle factors and reduced kidney function in older adults. We used the eGFR to evaluate changes in kidney function that may show reduced kidney function, but our objective was not to diagnose CKD. This was because cross-sectional studies using a secondary database cannot ensure whether individual participants had an eGFR of less than $60 \mathrm{ml} / \mathrm{min} / 1.73 \mathrm{~m}^{2}$ for more than 3 months, reflecting the guidelines for $\mathrm{CKD}$ diagnosis as described above. Among the important findings of our earlier study, older adults who had regular or occasional physical exercise had a significantly lower risk of reduced kidney function than those who did not exercise, while risk factors such as smoking, hypertension, obesity, and diabetes were significantly associated with a lower eGFR [10]. To both expand and confirm our previous results, and to further examine the influence of lifestyle factors on kidney function, the present study aimed to evaluate associations between lifestyle factors and reduced kidney function indicated by the eGFR levels in a large, nationwide sample of older adults in the United States.

\section{METHODS}

\section{Study Population}

The present study extracted cross-sectional data of older adults from the United States National Health and Nutrition Examination Survey (NHANES) to evaluate the associations between lifestyle factors and eGFR levels. NHANES is a continuous population-based database compiled in 2-year cycles by the National Center for Health Statistics (NCHS) of the United States Centers for Disease Control and Prevention (CDC), and the data are released every 2 years for research purposes. Since all NHANES data are de-identified, data analysis does not require Institutional Review Board approval or a signed informed consent from the participants.

To maximize the sample size, the data of participants from nine 2-year cycles of the NHANES (from 1999-2000 to 2015-2016) were combined and analyzed. The inclusion criteria were non-institutionalized older adults (aged 65 years and older). Participants with missing data for eGFR, physical activity, alcohol consumption, or cigarette smoking were excluded. Values for eGFR were calculated from serum creatinine standardized by isotope dilution mass spectrometry (IDMS) using the Chronic Kidney Disease Epidemiology Collaboration (CKD-EPI) formula [11]. An eGFR less than $60 \mathrm{ml} / \mathrm{min} / 1.73 \mathrm{~m}^{2}$ was defined as reduced kidney function; an eGFR $\geq 60 \mathrm{ml} / \mathrm{min} / 1.73 \mathrm{~m}^{2}$ was defined as normal kidney function, as previously described [10]. Because cross-sectional data of national surveys do not include longitudinal data and cannot confirm whether participants had eGFR values less than $60 \mathrm{ml} / \mathrm{min} / 1.73 \mathrm{~m}^{2}$ for more than 3 months, this study used "reduced kidney function" to describe participants with lower eGFR rather than suggesting CKD.

\section{Demographic Variables and Body Mass Index}

Age, sex, race, education level, and poverty income ratio were extracted from NHANES for all participants and analyzed. Race/ ethnicity was self-reported as Mexican American, Other Hispanic, Non-Hispanic White, Non-Hispanic Black, and Other Race/Ethnicity including multiracial. Education level was classified into three levels: with or without high school diploma and higher than high school. The family income-topoverty ratio ranges from 0 to 5 , with greater values indicating a higher income, and it was divided into three levels: $<1,1-3$, and $>3$. Body mass index (BMI) was classified into four categories-underweight $\left(\mathrm{BMI}<18.5 \mathrm{~kg} / \mathrm{m}^{2}\right)$, normal $(\mathrm{BMI}=$ $\left.18.5-24.9 \mathrm{~kg} / \mathrm{m}^{2}\right)$, overweight $\left(\mathrm{BMI}=25-29.9 \mathrm{~kg} / \mathrm{m}^{2}\right)$, and obese $\left(\mathrm{BMI}>30 \mathrm{~kg} / \mathrm{m}^{2}\right.$ ) - based on the criteria of the World Health Organization (WHO) (https://www.euro.who.int/en/healthtopics/disease-prevention/nutrition/a-healthy-lifestyle/bodymass-index-bmi).

\section{Lifestyle Factors}

The lifestyle factors examined in this study included physical activity, alcohol consumption, and cigarette smoking. In the NHANES database, leisure time physical activity was calculated by summing the product of weekly time spent in each activity multiplied by the metabolic equivalent of task (MET) value for that activity. Based on the MET scores, the participants were divided into three categories: inactive (0 MET$\mathrm{min} /$ week), insufficiently active (<750 MET-min/week), and sufficiently active ( $\geq 750 \mathrm{MET}-\mathrm{min} /$ week), as previously described [12].

The NHANES defined one alcohol-based drink as $12 \mathrm{oz}$. of beer, $4 \mathrm{oz}$. of wine, or an ounce of liquor. Participants who did not have at least 12 alcohol-based drinks in the past year or lifetime were classified as non-drinkers. Participants who had at least 12 drinks in their lifetime but not in the past year were classified as former drinkers. Participants who had at least 12 drinks in the past year and reported the number of drinks per week were recognized as current drinkers.

Participants who never had at least 100 cigarettes in their lifetime were classified as non-smokers. Participants who had at 
least 100 cigarettes but did not smoke now were classified as former smokers. Participants who had at least 100 cigarettes and reported the number of cigarettes per day in the past 30 days were classified as current smokers.

\section{Clinical Characteristics}

Blood levels of fasting glucose, total cholesterol, high-density lipoprotein (HDL), uric acid, blood urea nitrogen (BUN), and creatinine were obtained from the NHANES laboratory data. In addition, proteinuria was defined as urine albumin/creatinine ratio (UACR) $\geq 30 \mathrm{mg} / \mathrm{g}$.

The self-reported and the laboratory data of NHANES were utilized to identify participants with comorbidities. Cardiovascular diseases (coronary heart disease, angina pectoris, heart attack, stroke, and congestive heart failure) and cancer were defined by responding "yes" to any questions relative to previous diagnosis. Diabetes was defined as being told that they have diabetes or are at risk of diabetes, or taking insulin or antidiabetic medications. Hyperlipidemia was defined as being told that they have higher cholesterol level; are taking cholesterol-lowering medication; and/or having total cholesterol $>200 \mathrm{mg} / \mathrm{dl}$, triglyceride $>200 \mathrm{mg} / \mathrm{dl}$, or low-density lipoprotein (LDL) $>130 \mathrm{mg} / \mathrm{dl}$. Hyperuricemia was defined as being told that they have hyperuricemia or serum uric acid $>7 \mathrm{mg} / \mathrm{dl}$. Hypertension was defined as being told at least twice that they have high blood pressure, are taking antihypertensive medication, and/or having systolic blood pressure $\geq 140 \mathrm{mmHg}$ or diastolic blood pressure $\geq 90 \mathrm{mmHg}$. Urinary tract stones (renal calculi) were self-reported, but the corresponding NHANES questionnaire was only available from 2007 to 2016.

\section{Statistical Analysis}

Continuous variables are presented as the mean \pm standard error (SE), and differences were examined using the complex samples general linear model (CSGLM). Categorical variables are presented as counts and weighted percentages, and differences were tested by the Rao-Scott chi-square test. Interactions between covariates were evaluated. Univariate and multivariable regression models were constructed to identify factors associated with reduced kidney function. Significant interaction terms and significant variables identified in univariate analyses were used to establish the final multivariable models. Sensitivity analysis was performed to confirm the findings of the multivariable analysis, and subgroup analysis by sex was conducted. Special sample weights, stratum, and primary sampling units (PSUs) recommended by the NCHS were all included in the statistical analyses. All statistical analyses were performed using $\mathrm{SAS}^{\circledR}$ version 9.4 (Windows NT version, SAS Institute, Inc., Cary, NC, United States). A $p$-value $<0.05$ was considered statistically significant.

\section{RESULTS}

A total of 92,062 participants were included in the NHANES database from 1999 to 2016, of whom 12,551 participants were aged 65 years or older (Figure 1). After excluding participants

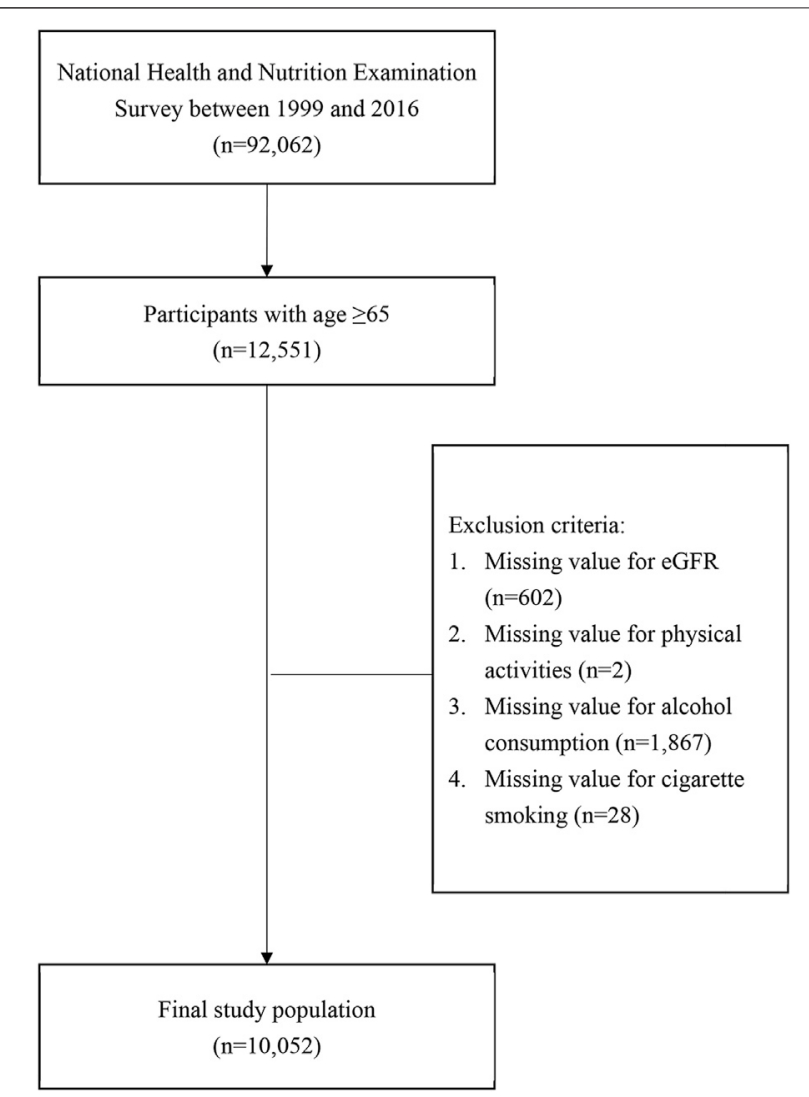

FIGURE 1 | Flowchart of study sample inclusion and exclusion criteria. (Associations between Lifestyle Factors and Reduced Kidney Function in US Older Adults: NHANES 1999-2016).

with missing values for eGFR and/or missing data for physical activity, alcohol consumption, or cigarette smoking, 10,052 participants were included as the analytic sample in the present study (Figure 1).

Eligible participants were stratified into two groups by eGFR level: the normal eGFR group (eGFR $\geq 60 \mathrm{ml} / \mathrm{min} / 1.73 \mathrm{~m}^{2}, n=$ $6,951)$ and the reduced eGFR group (eGFR $<60 \mathrm{ml} / \mathrm{min} / 1.73 \mathrm{~m}^{2}$, $n=3,101)$. The demographic, lifestyle-related, and clinical characteristics of the two eGFR groups are shown in Table 1. The mean ages were 72.27 and 75.77 years for participants with normal eGFR and reduced eGFR, respectively. The percentages of participants aged $65-74$ years were 66.23 and $40.28 \%$ in the normal and reduced eGFR groups, respectively. In addition to age, sex, race/ethnicity, education level, poverty/income ratio, BMI, physical activity, alcohol consumption, and cigarette smoking were significantly different between the two eGFR groups (all $p \leq 0.0001$; Table 1). Compared to the normal eGFR group, the reduced eGFR group had significantly higher blood levels of fasting glucose, total cholesterol, uric acid, BUN, and creatinine as well as a significantly higher percentage of proteinuria (all $p \leq 0.02$ ). However, the HDL blood levels were significantly lower in the reduced eGFR group than those in the normal eGFR group $(p=0.0005)$. Regarding comorbidities, the reduced eGFR group has significantly higher percentages of 
TABLE 1 | Demographic, lifestyle-related, and clinical characteristics of the study population stratified by eGFR levels. (Associations between Lifestyle Factors and Reduced Kidney Function in US Older Adults: NHANES 1999-2016).

\begin{tabular}{|c|c|c|c|}
\hline & eGFR $\geq 60$ & eGFR $<60$ & - \\
\hline & $n=6,951$ & $n=3,101$ & \\
\hline \multicolumn{4}{|l|}{ Demographics } \\
\hline Age, years & $72.27 \pm 0.10$ & $75.77 \pm 0.14$ & $<0.0001^{\mathrm{a}}$ \\
\hline $65-74$ & $4,357(66.23 \%)$ & $1,120(40.28 \%)$ & $<0.0001^{a}$ \\
\hline $75+$ & $2,594(33.77 \%)$ & 1981 (59.72\%) & \\
\hline \multicolumn{4}{|l|}{ Sex } \\
\hline Male & $3,608(46.48 \%)$ & $1,443(39.11 \%)$ & $<0.0001^{\mathrm{a}}$ \\
\hline Female & $3,343(53.52 \%)$ & $1,658(60.89 \%)$ & \\
\hline \multicolumn{4}{|l|}{ Race/ethnicity } \\
\hline Mexican american & 1,072 (3.87\%) & $264(2.3 \%)$ & $<0.0001^{\mathrm{a}}$ \\
\hline Other hispanic & $500(3.56 \%)$ & $150(2.57 \%)$ & \\
\hline Non-hispanic white & $3,875(81.07 \%)$ & $2,135(85.09 \%)$ & \\
\hline Non-hispanic black & $1,159(7.49 \%)$ & $429(6.62 \%)$ & \\
\hline Other-including multi-racial & $345(4 \%)$ & $123(3.43 \%)$ & \\
\hline \multicolumn{4}{|l|}{ Education level } \\
\hline Without high school diploma & $2,483(23.23 \%)$ & 1,107 (27.91\%) & $0.0001^{a}$ \\
\hline High school diploma & $1,610(25.60 \%)$ & $803(28.18 \%)$ & \\
\hline Higher than high school & $2,849(51.08 \%)$ & $1,183(42.75 \%)$ & \\
\hline Unknown/missing & $9(0.09 \%)$ & $8(0.16 \%)$ & \\
\hline \multicolumn{4}{|l|}{ Poverty/income ratio } \\
\hline$<1$ & 1,055 (8.97\%) & 447 (10.25\%) & $<0.0001^{\mathrm{a}}$ \\
\hline $1-3$ & $3,165(41.87 \%)$ & $1,544(48.36 \%)$ & \\
\hline$>3$ & 2070 (40.10\%) & 830 (33.03\%) & \\
\hline Unknown/missing & $661(9.07 \%)$ & $280(8.36 \%)$ & \\
\hline \multicolumn{4}{|l|}{$\mathrm{BMI}^{\mathrm{b}}$} \\
\hline Underweight & $104(1.62 \%)$ & 29 (0.98\%) & $<0.0001^{\mathrm{a}}$ \\
\hline Normal & 1847 (26.73\%) & 772 (25.57\%) & \\
\hline Overweight & 2,647 (37.67\%) & $1,121(35.61 \%)$ & \\
\hline Obesity & $2,194(31.99 \%)$ & $1,045(34.21 \%)$ & \\
\hline Unknown/missing & 159 (1.98\%) & $134(3.63 \%)$ & \\
\hline \multicolumn{4}{|l|}{ Lifestyle } \\
\hline Physical activity $(\mathrm{MET})^{\mathrm{C}}$ & $872.60 \pm 30.53$ & $582.29 \pm 42.62$ & $<0.0001^{a}$ \\
\hline Inactive & $3,262(43.1 \%)$ & 1823 (56.26\%) & $<0.0001^{a}$ \\
\hline Insufficiently active & $1,637(24.77 \%)$ & $640(22.48 \%)$ & \\
\hline Sufficiently active & 2052 (32.14\%) & $638(21.27 \%)$ & \\
\hline \multicolumn{4}{|l|}{ Alcohol consumption } \\
\hline No & $1,288(16.34 \%)$ & 699 (22.71\%) & $<0.0001^{a}$ \\
\hline Former drinker & $2,464(32.42 \%)$ & $1,293(38.3 \%)$ & \\
\hline Current drinker & $3,199(51.24 \%)$ & 1,109 (38.99\%) & \\
\hline \multicolumn{4}{|l|}{ Cigarette smoking } \\
\hline No & $3,301(47.56 \%)$ & 1,547 (51.6\%) & $<0.0001^{a}$ \\
\hline Former smoker & $2,890(42.64 \%)$ & $1,321(41.69 \%)$ & \\
\hline Current smoker & $760(9.81 \%)$ & $233(6.71 \%)$ & \\
\hline \multicolumn{4}{|l|}{ Blood examination } \\
\hline Fasting glucose, mg/dL & $113.02 \pm 0.77$ & $116.35 \pm 1.19$ & $0.02^{\mathrm{a}}$ \\
\hline Total cholesterol, mg/dL & $119.05 \pm 0.72$ & $192.94 \pm 1.06$ & $<0.0001^{a}$ \\
\hline $\mathrm{HDL}, \mathrm{mg} / \mathrm{dL}$ & $55.12 \pm 0.61$ & $51.26 \pm 0.77$ & $0.0005^{a}$ \\
\hline Uric acid, mg/dL & $5.35 \pm 0.02$ & $6.46 \pm 0.04$ & $<0.0001^{\mathrm{a}}$ \\
\hline $\mathrm{BUN}, \mathrm{mg} / \mathrm{dL}$ & $15.04 \pm 0.08$ & $22.80 \pm 0.20$ & $<0.0001^{a}$ \\
\hline Creatinine, $\mathrm{mg} / \mathrm{dL}$ & $0.85 \pm 0.003$ & $1.36 \pm 0.02$ & $<0.0001^{a}$ \\
\hline Proteinuria $^{d}$ & $1,422(18.15 \%)$ & $1,100(31.42 \%)$ & $<0.0001^{a}$ \\
\hline \multicolumn{4}{|l|}{ Comorbidities } \\
\hline Cardiovascular disease & $1,564(22.02 \%)$ & 1,237 (38.54\%) & $<0.0001^{a}$ \\
\hline Diabetes & $1,402(16.86 \%)$ & 890 (26.31\%) & $<0.0001^{a}$ \\
\hline Hyperlipidemia & $5,253(78.68 \%)$ & $2,296(76.34 \%)$ & $0.04^{\mathrm{a}}$ \\
\hline Hyperuricemia & $1,018(13.25 \%)$ & $1,182(36.09 \%)$ & $<0.0001^{a}$ \\
\hline Hypertension & $4,530(63.31 \%)$ & $2,424(77.51 \%)$ & $<0.0001^{a}$ \\
\hline Urinary tract stones ${ }^{\ominus}$ & $523(8.10 \%)$ & 259 (8.67\%) & 0.45 \\
\hline Cancer & $1,463(25.13 \%)$ & $800(28.83 \%)$ & $0.002^{\mathrm{a}}$ \\
\hline
\end{tabular}

Continuous variables are expressed as mean \pm standard error (SE) and tested by Complex Samples General Linear Model, while categorical variables are presented as counts (weighted percentage) and tested by Rao-Scott Chi-square test.

eGFR, estimated glomerular filtration rate; BMI, body mass index; MET, metabolic equivalent; HDL, high density lipoprotein; BUN, blood urea nitrogen.

a Statistical significance $\mathrm{p}<0.05$

${ }^{b}$ Underweight: $B M l<18.5 \mathrm{~kg} / \mathrm{m}^{2}$, normal: $18.5 \leq B M l<25.0 \mathrm{~kg} / \mathrm{m}^{2}$, overweight: $25.0 \leq B M l<30.0 \mathrm{~kg} / \mathrm{m}^{2}$, obesity: $B M l \geq 30 \mathrm{~kg} / \mathrm{m}^{2}$.

${ }^{c}$ Physical activity was defined by MET scores. Participants were divided into three MET groups: Inactive: 0 MET-min/week; Insufficiently active: <750 MET-min/week; Sufficiently active: $\geq 750 \mathrm{MET}$-min/week

${ }^{d}$ Urinary albumin-to-creatinine ratio $>3 \mathrm{mg} / \mathrm{mmol}$.

${ }^{e}$ The questionnaire used for defining kidney stones has been available since 2007 ( $n=4,653$ ). 
TABLE 2 | Significant interaction terms between covariates, and their associations with reduced kidney function. (Associations between Lifestyle Factors and Reduced Kidney Function in US Older Adults: NHANES 1999-2016).

\begin{tabular}{lc}
\hline Interaction & aOR (95\% Cl) \\
\hline Age $\times$ cardiovascular disease & $0.76(0.58-1.00)$ \\
Age $\times$ diabetes & $0.74(0.55-0.98)$ \\
Female $\times$ former drinker & $1.22(0.89-1.68)$ \\
Female $\times$ current drinker & $1.41(1.01-1.98)$ \\
Female x proteinuria & $0.72(0.56-0.94)$ \\
Female $\times$ hyperuricemia & $1.50(1.15-1.96)$ \\
Mexican american x proteinuria & $1.77(1.21-2.60)$ \\
Other hispanic x proteinuria & $1.58(0.72-3.45)$ \\
Non-hispanic black x proteinuria & $1.69(1.23-2.30)$ \\
Other racial x proteinuria & $1.14(0.58-2.27)$ \\
\hline
\end{tabular}

Multivariate model was adjusted for age, sex, race, physical activity, alcohol consumption, cigarette smoking, proteinuria, cardiovascular disease, diabetes, hyperlipidemia, hyperuricemia, hypertension, cancer, and interaction terms.

cardiovascular disease, diabetes, hyperuricemia, hypertension, and cancer than the normal eGFR group (all $p \leq 0.002$ ); however, the prevalence of hyperlipidemia was significantly higher in the normal eGFR group ( $p=0.04$; Table 1).

The significant interaction terms between covariates and their associations with reduced kidney function are presented in Table 2. After adjusting for the significant variables identified in the univariate logistic analysis and the significant interaction terms, multivariable logistic analysis revealed that older age [adjusted odds ratio $(\mathrm{aOR})=2.87,95 \% \mathrm{CI}=2.47-3.34)$, proteinuria $(\mathrm{aOR}=1.63,95 \% \mathrm{CI}=1.37-1.95)$, cardiovascular disease $(\mathrm{aOR}=1.87,95 \% \mathrm{CI}=1.55-2.27)$, diabetes $(\mathrm{aOR}=1.66$, $95 \% \mathrm{CI}=1.33-2.08)$, hyperuricemia $(\mathrm{aOR}=3.10,95 \% \mathrm{CI}=$ $2.57-3.73)$, and hypertension $(\mathrm{aOR}=1.38,95 \% \mathrm{CI}=1.21-1.57)$ were significantly associated with higher odds of having decreased kidney function (Table 3). In contrast, more recent survey cycle $(\mathrm{aOR}=0.97,95 \% \mathrm{CI}=0.95-1.00)$, Mexican American $(\mathrm{aOR}=0.44,95 \% \mathrm{CI}=0.33-0.59)$, Other Hispanic $(\mathrm{aOR}=0.66,95 \% \mathrm{CI}=0.45-0.98)$, Non-Hispanic Black $(\mathrm{aOR}=0.51,95 \% \mathrm{CI}=0.42-0.62)$, Other Race $(\mathrm{aOR}=$ $0.70,95 \%$ CI $=0.50-0.99)$, sufficient physical activity ( $\mathrm{aOR}=0.75,95 \% \mathrm{CI}=0.64-0.87$ ), current alcohol consumption $(\mathrm{aOR}=0.63,95 \% \mathrm{CI}=0.47-0.85)$, and being a current smoker $(\mathrm{aOR}$ $=0.76,95 \% \mathrm{CI}=0.61-0.96)$ were significantly associated with a lower risk of having decreased kidney function (Table 3). Furthermore, the results of the sensitivity analysis were consistent with the results of the multivariable logistic analysis, except for sex and diabetes (Table 3).

The distribution of alcohol drinks per week in participants with current alcohol consumption, including all current drinkers, those with reduced eGFR, and those with normal eGFR, is shown in Figure 2. The median (IQR) values were $0.97(0.23-3.87)$ and $0.99(0.24-4.81)$ for current alcohol drinkers with reduced eGFR and with normal eGFR, respectively (Figure 2 ).

After stratifying by sex, the associations between the covariates and reduced kidney function were evaluated (Table 4). Multivariable models were adjusted for significant variables identified in the univariate analyses and the significant interaction terms, but sex-related interaction terms were excluded in this subgroup analysis. The results revealed that males of older age $(\mathrm{aOR}=2.88,95 \% \mathrm{CI}=2.32-3.58)$, proteinuria $(\mathrm{aOR}=1.68,95 \% \mathrm{CI}=1.39-2.04)$, cardiovascular disease $(\mathrm{aOR}=1.91,95 \% \mathrm{CI}=1.49-2.45)$, diabetes $(\mathrm{aOR}=1.64$, $95 \% \mathrm{CI}=1.21-2.22)$, hyperuricemia $(\mathrm{aOR}=3.12,95 \% \mathrm{CI}=$ 2.61-3.81), and hypertension $(\mathrm{aOR}=1.36,95 \% \mathrm{CI}=1.13-1.61$ ) were significantly associated with higher odds of decreased kidney function. In contrast, males with current alcohol consumption $(\mathrm{aOR}=0.63,95 \% \mathrm{CI}=0.46-0.85)$ had significant lower odds of decreased kidney function (Table 4). On the other hand, females of older age ( $\mathrm{aOR}=2.89,95 \%$ $\mathrm{CI}=2.33-3.57)$, cardiovascular disease $(\mathrm{aOR}=1.83,95 \%$ $\mathrm{CI}=1.35-2.49)$, diabetes $(\mathrm{aOR}=1.69,95 \% \mathrm{CI}=1.27-2.25)$, and hyperuricemia $(\mathrm{aOR}=4.63,95 \% \mathrm{CI}=3.83-5.60)$ were significantly associated with higher odds of decreased kidney function. But females with sufficient physical activity $(\mathrm{aOR}=0.66,95 \% \mathrm{CI}=0.53-0.82)$ had significant lower odds of decreased kidney function (Table 4).

\section{DISCUSSION}

The main findings of the present cross-sectional study conducted among older adults in the United States NHANES database were that older age, proteinuria, cardiovascular disease, diabetes, hyperuricemia, and hypertension were significantly associated with higher odds of reduced kidney function, while sufficient physical activity, current alcohol consumption, and being a current smoker were significantly associated with lower odds of reduced kidney function. Furthermore, subgroup analysis by sex revealed several sex-differential associations. Firstly, proteinuria was a risk factor for reduced eGFR in males only. In contrast, sufficient physical activity served as a beneficial factor only in female participants, and only males could be benefited from current alcohol consumption. Also, the beneficial effect of being a current smoker was no longer significant in males or females.

The results of previous studies conducted in other populations report similar findings about the impact of exercise, alcohol consumption, smoking, and sex on the eGFR levels representing reduced kidney function in older adults. An elderly population-based study found that, among individuals with eGFR $<60$, only $3.5 \%$ were without renal damage or comorbidities associated with a risk of renal insufficiency, and those with eGFR > 60 had none of the associated risk factors for reduced kidney function [13]. The authors of that study also suggest that, although the eGFR in older adults has long been associated with aging itself, and that a low eGFR is also associated with incident frailty in the elderly population [14], the eGFR appears to be directly associated with CKD and comorbidities such as cardiovascular disease and hypertension [13]. Perhaps instead of screening for CKD using eGFR as an indicator of reduced kidney function, it may make more sense clinically to assess vascular health, which is shown to increase the risk of reduced kidney function in older adults [15].

Investigation of the Impact Goals of the American Health Association (AHA), which were developed as part of 
TABLE 3 | Associations between risk factors and reduced kidney function (eGFR $<60 \mathrm{ml} / \mathrm{min} / 1.73 \mathrm{~m}^{2}$ ) evaluated by logistic regression analysis. (Associations between Lifestyle Factors and Reduced Kidney Function in US Older Adults: NHANES 1999-2016).

\begin{tabular}{|c|c|c|c|}
\hline & \multirow{2}{*}{$\begin{array}{l}\text { Univariate } \\
\text { Or }(95 \% \mathrm{Cl})\end{array}$} & \multicolumn{2}{|c|}{ Multivariate $^{c}$} \\
\hline & & aOR (95\% CI) & $\beta \pm \mathrm{SE}$ \\
\hline Survey cycle & $0.97(0.95-1.00)$ & $0.97(0.95-1.00)$ & $0.41 \pm 0.11$ \\
\hline \multicolumn{4}{|l|}{ Demographics } \\
\hline \multicolumn{4}{|l|}{ Age, years } \\
\hline $65-74$ & Reference & Reference & Reference \\
\hline $75+$ & $2.91(2.61-3.24)$ & $2.87(2.47-3.34)$ & $-6.73 \pm 0.56$ \\
\hline \multicolumn{4}{|l|}{ Sex } \\
\hline Male & Reference & Reference & Reference \\
\hline Female & $1.35(1.21-1.51)$ & $1.25(0.92-1.69)$ & $-1.37 \pm 1.07$ \\
\hline \multicolumn{4}{|l|}{ Race/ethnicity } \\
\hline Mexican american & $0.57(0.48-0.67)$ & $0.44(0.33-0.59)$ & $8.33 \pm 0.96$ \\
\hline Other hispanic & $0.69(0.53-0.89)$ & $0.66(0.45-0.98)$ & $3.79 \pm 1.28$ \\
\hline Non-hispanic white & Reference & Reference & Reference \\
\hline Non-hispanic black & $0.84(0.74-0.96)$ & $0.51(0.42-0.62)$ & $8.24 \pm 0.79$ \\
\hline Other - including multi-racial & $0.82(0.64-1.04)$ & $0.70(0.50-0.99)$ & $3.14 \pm 1.08$ \\
\hline \multicolumn{4}{|l|}{ Education level } \\
\hline Without high school diploma & Reference & Reference & Reference \\
\hline High school diploma & $0.92(0.80-1.05)$ & $0.99(0.85-1.17)$ & $-0.73 \pm 0.64$ \\
\hline Higher than high school & $0.71(0.62-0.82)$ & $0.94(0.80-1.10)$ & $-0.16 \pm 0.65$ \\
\hline Unknown/missing & $1.59(0.52-4.88)$ & $2.01(0.64-6.35)$ & $-6.12 \pm 4.03$ \\
\hline \multicolumn{4}{|l|}{ Poverty/income ratio } \\
\hline$<1$ & Reference & Reference & Reference \\
\hline $1-3$ & $1.01(0.86-1.18)$ & $0.99(0.82-1.19)$ & $-1.23 \pm 0.76$ \\
\hline$>3$ & $0.72(0.60-0.86)$ & 1.05 (0.85-1.29) & $-1.12 \pm 0.65$ \\
\hline Unknown/missing & $0.81(0.65-1.00)$ & $0.91(0.73-1.14)$ & $-0.16 \pm 0.65$ \\
\hline \multicolumn{4}{|l|}{$\mathrm{BMl}$} \\
\hline Underweight & $0.63(0.37-1.09)$ & & \\
\hline Normal & Reference & - & - \\
\hline Overweight & $0.99(0.87-1.13)$ & & \\
\hline Obesity & $1.12(0.98-1.27)$ & & \\
\hline Unknown/missing & 1.91 (1.44-2.54) & & \\
\hline \multicolumn{4}{|l|}{ Lifestyle } \\
\hline \multicolumn{4}{|l|}{ Physical activity } \\
\hline Inactive & Reference & Reference & Reference \\
\hline Insufficiently active & $0.70(0.60-0.80)$ & $0.86(0.75-1.00)$ & $0.06 \pm 0.53$ \\
\hline Sufficiently active & $0.51(0.44-0.59)$ & $0.75(0.64-0.87)$ & $1.12 \pm 0.54$ \\
\hline \multicolumn{4}{|l|}{ Alcohol consumption } \\
\hline No & Reference & Reference & Reference \\
\hline Former drinker & $0.85(0.74-0.97)$ & $0.79(0.60-1.05)$ & $0.87 \pm 1.04$ \\
\hline Current drinker & $0.55(0.47-0.64)$ & $0.63(0.47-0.85)$ & $3.29 \pm 1.03$ \\
\hline \multicolumn{4}{|l|}{ Cigarette smoking } \\
\hline No & Reference & Reference & Reference \\
\hline Former smoker & $0.90(0.80-1.01)$ & $0.88(0.76-1.00)$ & $1.77 \pm 0.54$ \\
\hline Current smoker & $0.63(0.51-0.78)$ & $0.76(0.61-0.96)$ & $3.57 \pm 0.94$ \\
\hline \multicolumn{4}{|l|}{ Blood examination } \\
\hline Proteinuria $^{a}$ & $2.16(1.92-2.42)$ & 1.63 (1.37-1.95) & $-4.68 \pm 0.77$ \\
\hline \multicolumn{4}{|l|}{ Comorbidities } \\
\hline Cardiovascular disease & 2.22 (1.99-2.48) & $1.87(1.55-2.27)$ & $-4.89 \pm 0.80$ \\
\hline Diabetes & $1.76(1.50-2.06)$ & $1.66(1.33-2.08)$ & $-1.39 \pm 0.74$ \\
\hline Hyperlipidemia & $0.87(0.77-0.99)$ & $0.91(0.78-1.05)$ & $0.04 \pm 0.51$ \\
\hline Hyperuricemia & 3.70 (3.27-4.18) & $3.10(2.57-3.73)$ & $-9.69 \pm 0.71$ \\
\hline Hypertension & $2.00(1.77-2.25)$ & 1.38 (1.21-1.57) & $-2.65 \pm 0.50$ \\
\hline Urinary tract stones ${ }^{b}$ & $1.08(0.89-1.30)$ & - & - \\
\hline Cancer & $1.21(1.07-1.36)$ & $1.09(0.95-1.25)$ & $-0.36 \pm 0.54$ \\
\hline
\end{tabular}

OR: odds ratio; aOR: adjusted odds ratio; Cl: confidence interval; eGFR, estimated glomerular filtration rate; BMI, body mass index.

Significant values are in bold ( $\mathrm{p}<0.05)$.

a Urinary albumin-to-creatinine ratio $>3 \mathrm{mg} / \mathrm{mmol}$.

${ }^{b}$ The questionnaire used for defining kidney stones has been available since 2007 ( $n=4,653$ ).

${ }^{c}$ Multivariate model was adjusted for age, sex, race, physical activity, alcohol consumption, cigarette smoking, proteinuria, cardiovascular disease, diabetes, hyperlipidemia,

hyperuricemia, hypertension, cancer, and significant interaction terms. 


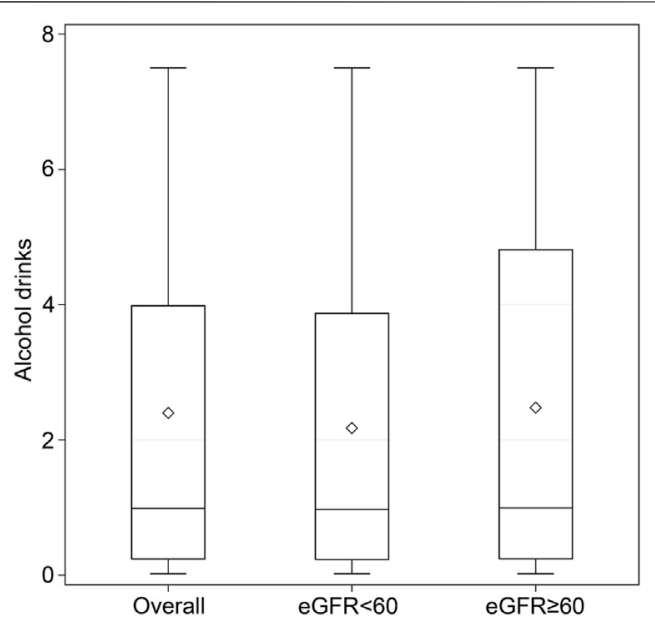

FIGURE 2 | Number of alcoholic drinks per week in participants with current alcohol consumption. There were 4,308 current drinkers, including 1,109 with eGFR $<60 \mathrm{ml} / \mathrm{min} / 1.73 \mathrm{~m}^{2}$ and 3,199 with eGFR $\geq 60 \mathrm{ml} / \mathrm{min} /$ $1.73 \mathrm{~m}^{2}$. Data are displayed as minimum, first quartile, third quartile, and maximum with a line at the median value and a diamond at the mean value in the box-and-whisker plot. (Associations between Lifestyle Factors and Reduced Kidney Function in US Older Adults: NHANES 1999-2016).

cardiovascular health promotion, for associations with risk of incident CKD showed that the AHA's "Life's Simple 7" promoting cardiovascular health simultaneously predicts a lower risk of CKD [16]. The Simple 7 factors are: nonsmoker or quit $>1$ year ago; $\mathrm{BMI}<25 \mathrm{~kg} / \mathrm{m}^{2} ; \geq 150 \mathrm{~min} /$ week of physical activity; healthy dietary habits; total cholesterol $<200 \mathrm{mg} / \mathrm{dl}$; blood pressure $<$ 120/80 mmHg; and fasting blood glucose < $100 \mathrm{mg} / \mathrm{dl}$ [16]. Smoking was also recently shown to be associated with a higher risk of developing CKD in healthy middle-aged Korean adults [17]. When the authors stratified their study population by age ( $<60 \mathrm{vs}$. $>60$ years), sex, smoking, diabetes, hypertension, and BMI, no significant interactions were found between the factors and the effects of current smoking on CKD [17].

In the present study, among the participants with current alcohol consumption, the majority were shown to consume four or fewer alcohol-based drinks per week, suggesting that mild to moderate alcohol was consumed by older participants. On the other hand, most participants with normal eGFR had five or fewer alcoholbased drinks per week. Importantly, the maximum number of alcohol drinks per week was less than eight in the present study, so it may be reasonable to assume that the included older adult participants consumed mild to moderate amounts of alcohol per week currently. In previous studies in the United States and Japan, mild to moderate alcohol consumption has been suggested to be associated with a lower risk of developing CKD [18, 19]. Hu et al. [18] conducted a prospective study of over 12,000 subjects in six alcohol consumption categories, finding, as we did, that drinking a moderate amount of alcohol may not harm the kidneys and is not associated with CKD based on an eGFR of $<60 \mathrm{ml} / \mathrm{min} / 1.73 \mathrm{~m}^{2}$. Matsumoto et al. [19] looked at both smoking and alcohol as risk factors contributing to $\mathrm{CKD}$, finding that mild to moderate alcohol consumption may be associated with CKD (i.e., low eGFR and proteinuria), but the same association was not shown in smokers; they concluded that the risk of
CKD may be less in smokers because smoking modifies the potential beneficial effect of alcohol in preventing CKD. The differences between the studies may be due to the different age groups studied.

In our previous study in a large Taiwanese population [10], male sex was significantly associated with an increased odds of having a reduced eGFR. Although the present NHANES study found that female older adults were about 1.25 times more likely to have reduced kidney function than are their male counterparts, such an association

TABLE 4 | Associations between risk factors and reduced kidney function (eGFR $<60 \mathrm{ml} / \mathrm{min} / 1.73 \mathrm{~m}^{2}$ ) by sex. (Associations between Lifestyle Factors and Reduced Kidney Function in US Older Adults: NHANES 1999-2016).

\begin{tabular}{|c|c|c|}
\hline & Male & Female \\
\hline & aOR $(95 \% \mathrm{Cl})$ & aOR (95\%Cl) \\
\hline Survey cycle & $0.97(0.94-1.01)$ & $0.97(0.94-1.01)$ \\
\hline \multicolumn{3}{|l|}{ Demographics } \\
\hline \multicolumn{3}{|l|}{ Age, years } \\
\hline $65-74$ & Reference & Reference \\
\hline $75+$ & $2.88(2.32-3.58)$ & $2.89(2.33-3.57)$ \\
\hline \multicolumn{3}{|l|}{ Race/ethnicity } \\
\hline Mexican american & $0.55(0.39-0.77)$ & $0.38(0.25-0.58)$ \\
\hline Other hispanic & $0.84(0.54-1.32)$ & $0.58(0.35-0.97)$ \\
\hline Non-hispanic white & Reference & Reference \\
\hline Non-hispanic black & $0.50(0.37-0.69)$ & $0.53(0.40-0.69)$ \\
\hline Other-including multi-racial & $0.52(0.29-0.94)$ & $0.87(0.54-1.41)$ \\
\hline \multicolumn{3}{|l|}{ Education level } \\
\hline Without high school diploma & Reference & Reference \\
\hline High school diploma & $0.81(0.63-1.04)$ & $1.13(0.91-1.41)$ \\
\hline Higher than high school & $0.85(0.67-1.08)$ & $1.00(0.81-1.22)$ \\
\hline Unknown/missing & $1.57(0.37-6.75)$ & $2.38(0.50-11.44)$ \\
\hline \multicolumn{3}{|l|}{ Poverty/income ratio } \\
\hline$<1$ & Reference & Reference \\
\hline $1-3$ & $0.91(0.70-1.19)$ & $1.03(0.81-1.32)$ \\
\hline$>3$ & $1.01(0.73-1.40)$ & $1.06(0.80-1.39)$ \\
\hline Unknown/missing & $0.93(0.65-1.33)$ & $0.90(0.65-1.23)$ \\
\hline \multicolumn{3}{|l|}{ Lifestyle } \\
\hline \multicolumn{3}{|l|}{ Physical activity } \\
\hline Inactive & Reference & Reference \\
\hline Insufficiently active & $0.92(0.73-1.15)$ & $0.83(0.69-1.00)$ \\
\hline Sufficiently active & $0.86(0.69-1.05)$ & $0.66(0.53-0.82)$ \\
\hline \multicolumn{3}{|l|}{ Alcohol consumption } \\
\hline No & Reference & Reference \\
\hline Former drinker & $0.80(0.60-1.08)$ & $0.96(0.80-1.15)$ \\
\hline Current drinker & $0.63(0.46-0.85)$ & $0.89(0.71-1.11)$ \\
\hline \multicolumn{3}{|l|}{ Cigarette smoking } \\
\hline No & Reference & Reference \\
\hline Former smoker & $0.87(0.72-1.04)$ & $0.88(0.74-1.05)$ \\
\hline Current smoker & $0.70(0.47-1.03)$ & $0.83(0.62-1.11)$ \\
\hline \multicolumn{3}{|l|}{ Blood examination } \\
\hline Proteinuria $a^{a}$ & $1.68(1.39-2.04)$ & $1.16(0.93-1.45)$ \\
\hline \multicolumn{3}{|l|}{ Comorbidities } \\
\hline Cardiovascular disease & $1.91(1.49-2.45)$ & $1.83(1.35-2.49)$ \\
\hline Diabetes & $1.64(1.21-2.22)$ & $1.69(1.27-2.25)$ \\
\hline Hyperlipidemia & $0.89(0.73-1.07)$ & $0.93(0.75-1.15)$ \\
\hline Hyperuricemia & $3.12(2.61-3.81)$ & $4.63(3.83-5.60)$ \\
\hline Hypertension & $1.36(1.13-1.61)$ & 1.39 (1.15-1.69) \\
\hline Cancer & $1.02(0.85-1.22)$ & $1.15(0.94-1.40)$ \\
\hline
\end{tabular}

OR: odds ratio; aOR: adjusted odds ratio; Cl: confidence interval; eGFR, estimated glomerular filtration rate.

Significant values are in bold $(\mathrm{p}<0.05)$.

a Urinary albumin-to-creatinine ratio $>3 \mathrm{mg} / \mathrm{mmol}$.

${ }^{b}$ Multivariate model was adjusted for survey cycle, age, sex, race, physical activity, alcohol consumption, cigarette smoking, proteinuria, cardiovascular disease, diabetes, hyperlipidemia, hyperuricemia, hypertension, cancer, and significant interaction terms (except for sex-related interaction terms). 
did not reach a significant level. In seeking to explain the gender discrepancies between that study and the present study, we stratified all participants by sex and re-evaluated the associations between life factors and reduced kidney function. Almost all of the risk factors identified in the population were still valid in either male or female participants, except proteinuria that was significant in males only. In addition, differences in the beneficial factors between males and females were also observed. Males and females benefited from current alcohol consumption and sufficient physical activity, respectively. Surprisingly, after stratifying by sex, being a current smoker was no longer a beneficial factor for reduced kidney function in males and females. The differences between the studies may be partially explained, as in Carrero et al. [20], by the greater prevalence of $\mathrm{CKD}$ in women and the tendency of longer life among women; thus, in study populations, we must consider the effects of longer life on natural declines in the eGFR. The authors also pointed out the potential overdiagnosis of $\mathrm{CKD}$ through the inappropriate use of eGFR equations [20]. Akasaka et al. [21] also emphasized the influence of elevated uric acid levels, characteristic of a reduced kidney function, on both eGFR and gender differences; uric acid concentration appears to accelerate the decline in eGFR values, and women appear to be more susceptible to urate-induced eGFR decline.

In the present study, physical exercise as reported in the NHANES database was measured in METs, which converts frequency and duration to minutes of activity per week, weighted by multiplying by an estimated MET value using the Compendium of Physical Activity values for intensity assessment [15] and summed across all activities to total physical activity. The MET results showed that participants with sufficient physical activity had significantly lower odds of having decreased kidney function. In our previous cross-sectional study [10], which analyzed data from the Taipei City Elderly Health Examination database 2006-2012, the results showed that participants who had regular or occasional exercise had significantly lower risks of having reduced kidney function than those without exercise.

The effects of home-based exercise therapy on kidney function in pre-dialysis CKD patients have also been explored previously [22], demonstrating that home-based exercise therapy did not affect kidney function in pre-dialysis CKD patients, even though it helped to improve leg muscle strength. A study that examined the eGFR levels in community-based older adults after a 12-week exercise program and post-exercise protein supplementation had modest positive effects and did not show a deleterious effect on kidney function, but the endpoint eGFR levels of the combined intervention were not significantly different before and after [23]. A cross-sectional study of the association between physical activity and kidney function in older men reported that higher levels of physical activity were associated with reduced odds of a lower eGFR, while each added $30 \mathrm{~min}$ of sedentary behavior per day was associated with greater odds of a lower eGFR [24]. Even without knowing whether the associations are causal, they were projected to be of public health concern in men with other risk factors for CKD.

\section{Strengths and Limitations}

The present study was strengthened by the use of data from nine 2year cycles of the NHANES database 1999-2016, a continuous crosssectional health survey that collects and analyzes data representative of the national United States non-institutionalized population. Because we included over 10,000 older adults with eGFR values, the sample size is large enough for fairly precise prevalence measures at the national level. Conducting this study using a nationally representative sample allows our results to be generalized across the entire United States older adult population. Nevertheless, certain limitations must also be noted, including that it involved crosssectional analysis, which precludes making inferences about causality. In addition, the NHANES data are from interviews and self-reported questionnaires and may be subject to recall problems or misunderstanding of the questions, which may reduce the accuracy of the data to some extent. The NHANES database also includes only United States data, including representative portions of different ethnic populations, and may not support conclusions about racial/ethnic factors or any populations outside of the United States. Further studies are needed to validate these findings in other populations and countries.

\section{Conclusion}

The present study identified several risk and beneficial factors for reduced kidney function in adults aged 65 and above and found that some risk and beneficial factors might be sex-specific. These findings may be useful in developing strategies for public health policies regarding risk and beneficial factors associated with reduced kidney function in older adults, as well as helping to explore whether screening using eGFR may be of value toward the early diagnosis of reduced kidney function in high-risk older adult populations.

\section{ETHICS STATEMENT}

Ethical approval was not provided for this study on human participants because this study conducted secondary analysis of cross-sectional data of older adults derived from the United States National Health and Nutrition Examination Survey (NHANES). Since all NHANES data are de-identified, data analysis does not require IRB approval or signed informed consent of the participants. Written informed consent for participation was not required for this study in accordance with the national legislation and the institutional requirements.

\section{AUTHOR CONTRIBUTIONS}

Conception and study design by all authors; data acquisition by $\mathrm{H}-\mathrm{JC}$ and K-RL; data analysis and interpretation by $\mathrm{H}-\mathrm{JC}$ and K-RL; statistical analysis by $\mathrm{H}-\mathrm{JC}$ and K-RL; manuscript drafted by K-RL and M-TL; critical revision of the manuscript by $\mathrm{H}-\mathrm{JC}$ and J-LC; all authors final approved the manuscript.

\section{CONFLICT OF INTEREST}

The authors declare that the research was conducted in the absence of any commercial or financial relationships that could be construed as a potential conflict of interest. 


\section{REFERENCES}

1. Abdelhafiz, AH, Brown, SHM, Bello, A, and El Nahas, M. Chronic Kidney Disease in Older People: Physiology, Pathology or Both? Nephron Clin Pract (2010). 116:c19-c24. doi:10.1159/000314545

2. Beard, JR, Officer, A, de Carvalho, IA, Sadana, R, Pot, AM, and Michel, JP. The World Report on Ageing and Health: a Policy Framework for Healthy Ageing. The Lancet (2016). 387:2145-54. doi:10.1016/S0140-6736(15) 00516-4

3. Grundy, SM. Age as a Risk Factor: You Are as Old as Your Arteries. Am J Cardiol (1999). 83:1455-A7. doi:10.1016/s0002-9149(99)00125-3

4. Bleyer, AJ, Shemanski, LR, Burke, GL, Hansen, KJ, and Appel, RG. Tobacco, Hypertension, and Vascular Disease: Risk Factors for Renal Functional Decline in an Older Population. Kidney Int (2000). 57:2072-9. doi:10.1046/j.15231755.2000.00056.x

5. Winearls, CG, and Glassock, RJ. Dissecting and Refining the Staging of Chronic Kidney Disease. Kidney Int (2009). 75:1009-14. doi:10.1038/ ki.2009.49

6. United States Renal Data System. 2016 Annual data reportchapter 1: CKD in the general population. Available from: https:/www.usrds.org/2016/view/v1_ 01.aspx (Accessed Nov. 19, 2020).

7. Hill, NR, Fatoba, ST, Oke, JL, Hirst, JA, O'Callaghan, CA, and Lasserson, DS. Global Prevalence of Chronic Kidney Disease - A Systematic Review and Meta-Analysis. PLoS One (2016). 11:e0158765. doi:10.1371/ journal.pone.0158765

8. Gaitonde, DY, Cook, DL, and Rivera, IM. Chronic Kidney Disease: Detection and Evaluation. Am Fam Physician (2017). 96:776-83.

9. Kazancioglu, R. Risk Factors for Chronic Kidney Disease: an Update. Kidney Int Suppl (2011). 3:368-71. doi:10.1038/kisup.2013.79

10. Chang, H-J, Lin, K-R, Lin, M-T, and Chang, J-L. Association between Lifestyle Factors and Decreased Kidney Function in Older Adults: a Community-Based Cross-Sectional Analysis of the Taipei City Elderly Health Examination Database. BMC Nephrol (2020). 21:169. doi:10.1186/s12882-020-01838-1

11. Matsushita, K, Mahmoodi, BK, Woodward, M, Emberson, JR, Jafar, TH, and Jee, SH. Comparison of Risk Prediction Using the CKD-EPI Equation and the MDRD Study Equation for Estimated Glomerular Filtration Rate. JAMA (2012). 307:1941-51. doi:10.1001/jama.2012.3954

12. Orces, CH. Association between Leisure-Time Aerobic Physical Activity and Vitamin D Concentrations Among US Older Adults: the NHANES 2007-2012. Aging Clin Exp Res (2019). 31:685-93. doi:10.1007/s40520-018-1031-9

13. Abdulkader, RCRM, Burdmann, EA, Lebrão, ML, Duarte, YAO, and Zanetta, DMT. Aging and Decreased Glomerular Filtration Rate: An Elderly Population-Based Study. PLoS One (2017). 12:e0189935. doi:10.1371/ journal.pone.0189935

14. Guerville, F, de Souto Barreto, P, Taton, B, Bourdel-Marchasson, I, Rolland, Y, and Vellas, B. Estimated Glomerular Filtration Rate Decline and Incident Frailty in Older Adults. Cjasn (2019). 14:1597-604. doi:10.2215/CJN.03750319
15. DeFina, LF, Radford, NB, Barlow, CE, Willis, BL, Leonard, D, and Haskell, WL. Association of All-Cause and Cardiovascular Mortality with High Levels of Physical Activity and Concurrent Coronary Artery Calcification. JAMA Cardiol (2019). 4:174-81. doi:10.1001/jamacardio.2018.4628

16. Rebholz, CM, Anderson, CAM, Grams, ME, Bazzano, LA, Crews, DC, and Chang, AR. Relationship of the American Heart Association's Impact Goals (Life's Simple 7) with Risk of Chronic Kidney Disease: Results from the Atherosclerosis Risk in Communities (ARIC) Cohort Study. Jaha (2016). 5: e003192. doi:10.1161/JAHA.116.003192

17. Jo, W, Lee, S, Joo, YS, Nam, KH, Yun, H-R, and Chang, TI. Association of Smoking with Incident CKD Risk in the General Population: A CommunityBased Cohort Study. PLoS One (2020). 15:e0238111. doi:10.1371/ journal.pone.0238111

18. Hu, EA, Lazo, M, Rosenberg, SD, Grams, ME, Steffen, LM, and Coresh, J. Alcohol Consumption and Incident Kidney Disease: Results from the Atherosclerosis Risk in Communities Study. J Ren Nutr (2020). 30:22-30. doi:10.1053/j.jrn.2019.01.011

19. Matsumoto, A, Nagasawa, Y, Yamamoto, R, Shinzawa, M, Hasuike, Y, and Kuragano, T. The Association of Alcohol and Smoking with CKD in a Japanese Nationwide Cross-Sectional Survey. Hypertens Res (2017). 40:771-8. doi:10.1038/hr.2017.25

20. Carrero, JJ, Hecking, M, Chesnaye, NC, and Jager, KJ. Sex and Gender Disparities in the Epidemiology and Outcomes of Chronic Kidney Disease. Nat Rev Nephrol (2018). 14:151-64. doi:10.1038/nrneph.2017.181

21. Akasaka, H, Yoshida, H, Takizawa, H, Hanawa, N, Tobisawa, T, and Tanaka, M. The Impact of Elevation of Serum Uric Acid Level on the Natural History of Glomerular Filtration Rate (GFR) and its Sex Difference. Nephrol Dial Transplant (2014). 29:1932-9. doi:10.1093/ndt/gfu197

22. Hiraki, K, Shibagaki, Y, Izawa, KP, Hotta, C, Wakamiya, A, and Sakurada, T. Effects of home-based Exercise on Pre-dialysis Chronic Kidney Disease Patients: a Randomized Pilot and Feasibility Trial. BMC Nephrol (2017). 18:198. doi:10.1186/s12882-017-0613-7

23. Ramel, A, Arnarson, A, Geirsdottir, OG, Jonsson, PV, and Thorsdottir, I. Glomerular Filtration Rate after a 12-wk Resistance Exercise Program with post-exercise Protein Ingestion in Community Dwelling Elderly. Nutrition (2013). 29:719-23. doi:10.1016/j.nut.2012.10.002

24. Parsons, TJ, Sartini, C, Ash, S, Lennon, LT, Wannamethee, SG, and Lee, IM. Objectively Measured Physical Activity and Kidney Function in Older Men; a Cross-Sectional Population-Based Study. Age Ageing (2017). 46:1010-4. doi:10.1093/ageing/afx091

Copyright (c) 2021 Chang, Lin, Lin and Chang. This is an open-access article distributed under the terms of the Creative Commons Attribution License (CC BY). The use, distribution or reproduction in other forums is permitted, provided the original author(s) and the copyright owner(s) are credited and that the original publication in this journal is cited, in accordance with accepted academic practice. No use, distribution or reproduction is permitted which does not comply with these terms. 